\title{
HIGGS FACTORY AND POTENTIALS
}

\author{
Z. Parsa*, Brookhaven National Laboratory, Physics Dept., 510 A, Upton, NY 11973, USA.
}

\begin{abstract}
Recent experimental results from LEP has generated new interest in potentials of a Higgs factory (as the first Muon collider). We present a conceptual overview of such a collider, required Luminosity, and some physics potentials. We illustrate the unlikely case where the muon radiative corrections results in a vanishing $\mu^{+} \mu^{-} h$ vertex ( $\sigma$ is zero), and how in such a case, the Neutral MSSM Higgs sector via resonant production of $\mathrm{H}$ and $\mathrm{A}$ may be possible at a muon collider.
\end{abstract}

\section{INTRODUCTION}

Finding Higgs bosons, thus understanding the origin of electroweak symmetry breaking, supersymmetric (SUSY) particles, and more are some of the reasons for high energy physics community's interest in colliders beyond the $e^{+} e^{-}$ colliders and LHC.

In devising a strategy for technical accelerator possibilities, we note that the experiments over the last two decades have convincingly shown that the strong, electro-magnetic, and weak forces are all closely related and are simply described by the "Standard Model." Although there is now little doubt that the Standard Model is a very good description of the basic forces responsible for all atomic and nuclear physics, there remain many open questions [?, 3], and new physics beyond what has been observed is required. The simplest possibility, the "Higgs Mechanism" predicts the existence of a fundamental Higgs Boson. Finding that elusive particle or whatever new physics is actually responsible for mass generation remains the primary goal of the next generation of colliders.

Noting that, recently Experiment E821 at Brookhaven National Laboratory announced that, a precision measurement of the anomalous magnetic moment of the muon, deviates by a 2.6 sigma from the value predicted by the Standard Model (after analysis of their 1999 data). This could indicate that other physical theories that go beyond the assumptions of the Standard Model may now be open to experimental exploration. E.g., that 2.6 sigma difference from the Standard Model may be a strong indication of supersymmetry in roughly the $\tan \beta \simeq 4, m_{\mathrm{SUSY}} \simeq 100$ $\mathrm{GeV}-\tan \beta \simeq 40, m_{\mathrm{SUSY}} \simeq 450 \mathrm{GeV}$ region or an indication of radiative muon mass generation from "new physics" in the $1-2 \mathrm{TeV}$ range. Both cases can illustrate exciting prospects with very interesting implications for future experiments. Obviously, before the assertion of any "new physics" can be taken seriously, one has to wait

\footnotetext{
* Supported by US Department of Energy contract Number DE-AC0298CH10886. E-mail: parsa@bnl.gov
}

for completion of the E821 (2000 and 2001 data) analysis, (which has about 7 times the amount of data that was already reported in 1999), and further checks on refining the values of $a_{\mu}^{\exp }$ and $a_{\mu}^{\mathrm{SM}}$ [3].

\section{PHYSICS AT FUTURE COLLIDERS?}

The high energy community is interested in the potential of colliders beyond the $\left(e^{+} e^{-}\right)$colliders and LHC. Particle beam colliders are the primary tools for performing high energy physics research. Collisions of high energy particles produce events in which much of the energy of the beams can be converted into the masses of new heavy particles not normally found in nature. By studying the production and decay of these new particles, the underlying structure of the universe and the laws that govern it are unveiled.

The Higgs mass region of $110 \sim 130 \mathrm{GeV}$ or higher may be explored via $W^{ \pm} H$ and $Z H$ associated production if the $H \rightarrow b \bar{b}$ mode is resolvable.

Asymmetric $B$ factories provide new ways to explore $\mathrm{CP}$ violation. LEPII has achieved $e^{+} e^{-}$center-of-mass energy of about $\gtrsim 207 \mathrm{GeV}$, (e.g., ALEPH collected $217 p b^{-1}$ of data at energies 200-209 GeV in 2000) and a mass bound of $\gtrsim 113 \mathrm{GeV}$ on Higgs boson mass. Furthermore, the $W^{ \pm}$ mass has been measured at LEPII, and Fermilab, providing an interesting constraint on the Higgs mass via quantum loop relations.

In some longer term $(\sim 2006)$, the LHC $p p$ collider with $\sqrt{s}=14 \mathrm{TeV}$ should find the Higgs scalar or tell us it doesn't exist. If SUSY exists $\lesssim 1 \mathrm{TeV}$, it will be discovered. Hopefully, completely unexpected revelations will also be made. Beyond the LHC, various collider options are possible. The Next Linear Collider (NLC) may start $e^{+} e^{-}$collisions at $\sqrt{s}=500 \mathrm{GeV}$ and be upgradeable to $1-1.5 \mathrm{TeV}$. It would have high luminosity $>5 \times 10^{33}$ and polarization. The NLC also offers $\gamma \gamma, e^{-} e^{-}$, and $e^{-} \gamma$ collider options which expand its physics potential. There has been also some discussion of possible future $e^{+} e^{-}$colliders with $\sqrt{s} \simeq 5 \mathrm{TeV}$, a major step, if achievable. The NLC will be a superb tool for studying the Higgs, SUSY, Technicolor etc., [4].

Other possibilities include a $\mu^{+} \mu^{-}$collider which has a very interesting concept, but require series studies and technology demonstrations. Various machine energies has been considered previously by our collaboration [5]. Table 1, illustrates some parameters.

\section{MUON COLLIDER}

Fig. 1 shows a schematic of a muon collider components [5], and how the accelerator ring may compactly contain the other components. A high intensity proton source is bunch compressed and focused on a heavy metal target. 
Table 1: Example of Parameter Sets For Muon Colliders

\begin{tabular}{|c|c|c|}
\hline $\begin{array}{c}\text { center of mass energy, } \mathrm{E}_{\mathrm{CoM}} \\
\text { description (M.C.) }\end{array}$ & $\begin{array}{l}0.1 \text { to } 3 \mathrm{TeV} \\
\text { Higgs to H.E. }\end{array}$ & $\begin{array}{c}400 \mathrm{GeV} \\
\text { Top threshold }\end{array}$ \\
\hline \\
\hline Luminosity, $\mathcal{L}\left[10^{32} \mathrm{~cm}^{-2} . \mathrm{s}^{-1}\right]$ & $(0.08 \rightarrow 700)$ & 30 \\
\hline $\mathcal{L} \mathrm{dt}\left[\mathrm{fb}^{-1} /\right.$ year $]$ & $0.08 \rightarrow 700$ & 30 \\
\hline No. of $\mu \mu \rightarrow$ ee events/det/year & $650 \rightarrow 13000$ & 16000 \\
\hline No. of (115 GeV) SM Higgs/year & $2000 \rightarrow 800000$ & 14000 \\
\hline C.M energy spread, $\sigma_{\mathrm{E}} / \mathrm{E}\left[10^{-3}\right]$ & $0.02 \rightarrow 1.1$ & 1.4 \\
\hline \multicolumn{3}{|l|}{ collider ring parameters: } \\
\hline circumference, $C[\mathrm{~km}]$ & $0.35 \rightarrow 6.0$ & 1.0 \\
\hline ave. bending B field $[\mathrm{T}]$ & $3.0 \rightarrow 5.2$ & 4.2 \\
\hline \multicolumn{3}{|l|}{ beam parameters: } \\
\hline ( $\mu^{-}$or) $\mu^{+} /$bunch, $\mathrm{N}_{0}\left[10^{12}\right]$ & $2.0 \rightarrow 4.0$ & 4.0 \\
\hline$\left(\mu^{-}\right.$or) $\mu^{+}$bunch rep. rate, $\mathrm{f}_{\mathrm{b}}[\mathrm{Hz}]$ & $15 \rightarrow 30$ & \\
\hline 6-dim. norm. emit., $\epsilon_{6 N}\left[10^{-12} \mathrm{~m}^{3}\right]$ & $170 \rightarrow 170$ & 170 \\
\hline $\mathrm{x}, \mathrm{y}$ norm. emitt. $[\pi . \mathrm{mm} . \mathrm{mrad}]$ & $50 \rightarrow 290$ & 77 \\
\hline Long. emitt. $\left[10^{-3} \mathrm{eV} . \mathrm{s}\right]$ & $0.81 \rightarrow 24$ & 10 \\
\hline Fract. mom. spread, $\delta\left[10^{-3}\right]$ & $0.030 \rightarrow 1.6$ & 2.0 \\
\hline
\end{tabular}

The pions generated are captured by a high field solenoid and transferred to solenoidal decay channel within a low frequency linac. The linac reduces, by phase rotation the momentum spread of the pions and of the muons into which they decay. Subsequently, the muons are cooled by a sequence of ionization cooling stages, and must be rapidly accelerated to avoid decay. This can be done in recirculating accelerators (as at CEBAF) or in fast pulsed synchrotrons. Muon collisions occur in a separate high field collider storage ring with a single very low beta insertion. A muon collider with center of mass energy less than about

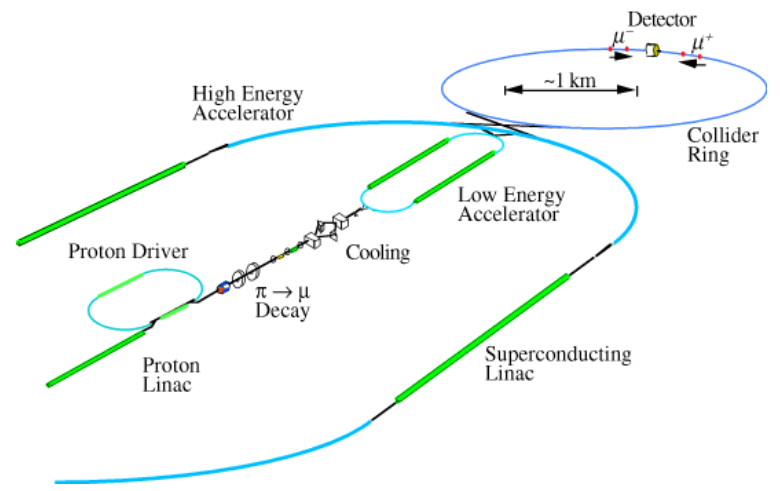

Figure 1: Schematic of How the accelerator ring may compactly contain the other components

$10 \mathrm{TeV}$ can be circular, and can tolerate a far larger spot size than an electron linear collider since the muons make about 1000 crossings. Muon $\left(m_{\mu} / m_{e}=207\right)$ have the same advantage in energy reach as electron but has little beamstrahlung, thus very small energy spread is obtainable. In addition the direct coupling of lepton-lepton system to Higgs boson has a cross section proportional to the square of the lepton mass. thus the cross section for direct Higgs production from a $\mu^{+} \mu^{-}$collider is about 40,000 times that from an $e^{+} e^{-}$collider system.

A large effort previously was devoted to design and assessing the feasibility of building a high energy muon col- lider. Unfortunately, the estimates of the cost and radiation problems, etc., seems to prohibit muon colliders at the high energies, without some drastic new developments? Since building a full high energy Muon Collider will take a considerable time to realize, as a first step a Muon storage ring based Neutrino Factory has been considered by our collaboration[1,9]. To upgrade for e.g., the $20 \mathrm{GeV}$ BNLsite specific [9] neutrino factory facility presented in [1] it would require some changes in the front end, cooling, acceleration and replacing of the storage ring by a collider ring (shown in Fig. 1). The cooling is the major components that has not yet been demonstrated. For example of a beam from target with rms (transverse and longitudinal) emittances of $2 \times 10^{-2}$ and $1 \mathrm{~m}$-rad respectively, the muon collider, (of $10^{12} \mu /$ bunch), rms transverse and longitudinal emittances are $10^{-4}$ and $10^{-2} \mathrm{~m}$-rad respectively. That is it would require a 6-Dimentional cooling of about $4 \times 10^{6}$. Starting with the same beam from target (as above) for the neutrino factory, the rms transverse and longitudinal emittances are about $2 \times 10^{-3}$ and $10^{-2} \mathrm{~m}$-rad respectively (100 bunches), and need cooling of about $10^{2}$, which is about $10^{4}$ less cooling as compared to the requirement for the muon collider $[5,9]$.

\section{LUMINOSITY AND PHYSICS POTENTIALS}

Muon Colliders have unique physics and technical advantages and disadvantages as compared to $e^{+} e^{-}$and hadron colliders and are to be considered as complementary. For the same energy and integrated luminosity, anything that can be done at $e^{+} e^{-}$, should be possible at $\mu^{+} \mu^{-}$ collider, and more. E.g., possibilities for s-channel Higgs production, and a higher center-of mass energy with reduced backgrounds. Both of which are due to the large muon mass as compared to the electron mass. Higher energy may be crucial e.g., in improving signals for WW scattering, and the kinematical reach for pair production of SUSY particles.

The figure of merit in physics searches at an $e^{+} e^{-}$or $\mu^{+} \mu^{-}$collider, is expressed by the QED point cross section for $e^{+} e^{-} \rightarrow \mu^{+} \mu^{-}$:

$$
\sigma_{Q E D}(\sqrt{s})=\left(\frac{100[f b]}{s\left[T_{e} V^{2}\right]}\right) \times\left(\frac{\alpha(s)}{\alpha\left(M_{z}^{2}\right)}\right)
$$

As before e.g., [6], we will neglect the factor $\left(\frac{\alpha(s)}{\alpha\left(M_{z}^{2}\right)}\right)$ as it varies slowly with $\mathrm{s}$. If the integrated luminosity needed for studying the new physics signals is:

$$
\left(\int L d t\right) \sigma_{Q E D} \gtrsim 1000 \text { events, }
$$

then the $\mu^{+} \mu^{-}$collider design should be able to deliver an integrated luminosity of

$$
\left(\int L d t\right) \gtrsim 10 s\left[f b^{-1}\right]
$$

If this is to be accumulated for one year of running time, the required luminosity estimate is

$$
L\left[\mathrm{fb}^{-1}\right] \gtrsim 10^{33} \mathrm{~s}\left[\mathrm{~cm}^{-2}\right]\left[\mathrm{sec}^{-1}\right] .
$$


E.g., The estimates of the luminosity requirements for a

$$
\text { - For } \sqrt{s} \simeq 0.1[\mathrm{TeV}], L\left[\mathrm{fb}^{-1}\right] \gtrsim 10^{31}\left[\mathrm{~cm}^{-2}\right]\left[\mathrm{sec}^{-1}\right] \text {. }
$$

\subsection{Higgs Propagator \& Radiative Corrections}

In the minimal supersymetric extension of standard model (MSSM), there are 3 neutral Higgs bosons, two scalars (h and $\mathrm{H}$ ) and one pseudo-scalar (A). A muon collider may provide unique opportunities for measuring the Higgs width, various $\mathrm{CP}$-violating observables and to study the heavier neutral Higgs bosons $\mathrm{H}$, A.

Here we consider although unlikely, the case where the cross section $\left.\sigma\left(\mu^{+} \mu^{-} \rightarrow h\right) \rightarrow 0\right)$, i.e. where the muon radiative corrections results in a vanishing $\mu^{+} \mu^{-} h$ vertex. Where would this happen? What happends then with the heavy Higgs (how are they different)?, etc. Figs. 2-4 illustrates the above possibilities and the importance of inclusion of the radiative corrections. For these examples (in MSSM) the $M_{A}-\tan \beta$ parameter space was scaned and 1) a low SUSY mass scale $M_{S U S Y}=300 \mathrm{GeV}$, or 2 ) a high scale $M_{S U S Y}=1000 \mathrm{GeV}$ was used. The other parameters were chosen to be $\mu, X_{t}= \pm M_{S U S Y}$ or $\pm 2 M_{S U S Y}$ (with $X_{t}=A_{t}-\mu \cot \beta$ being the off-diagonal entry in the $\tilde{t}$ mass matrix.) The plots in Figs. 2, 3 show where the cross sections would be zero. The region for one special case with $M_{S U S Y}=300 \mathrm{GeV}$ where the cross section is reduced to $<30 \%$ is shown in Fig. 4. [8]. The alternative

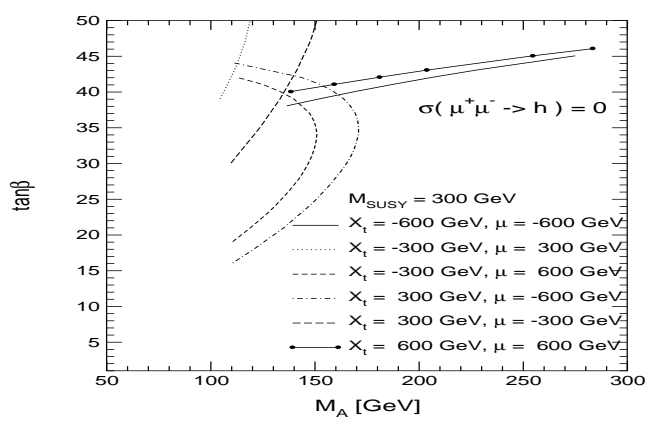

Figure 2: Regions in the $M_{A}-\tan \beta$ plane where $\sigma(\mu \mu \rightarrow$ $h)=0$ for various values of $M_{S U S Y}=300 \mathrm{GeV}$.

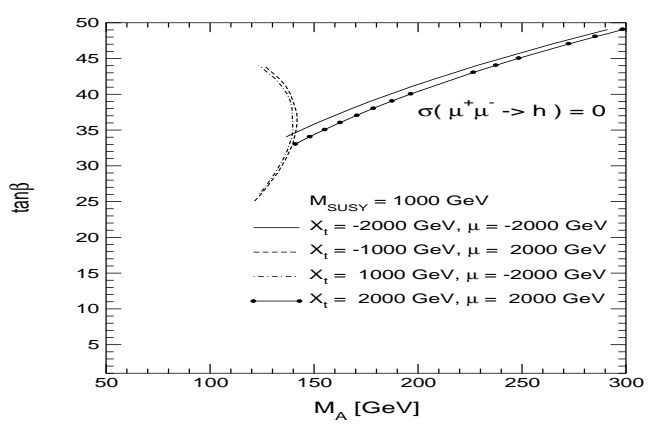

Figure 3: Regions in the $M_{A}-\tan \beta$ plane where $\sigma(\mu \mu \rightarrow$ $h)=0$ for various values of $M_{S U S Y}=1000 \mathrm{GeV}$.

would be to investigate Heavy neutral Higgs bosons where the masses are in the 100 to $300 \mathrm{GeV}$ range and often the

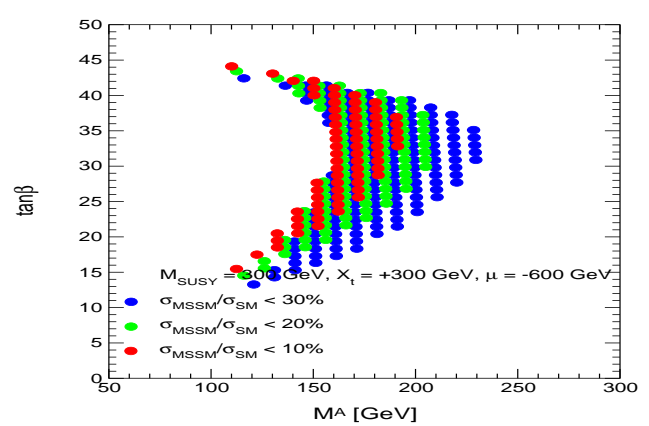

Figure 4: Regions in the $M_{A}-\tan \beta$ plane where $\sigma(\mu \mu \rightarrow$ $h)=0$ for various values of $M_{S U S Y}=1000 \mathrm{GeV}$.

mass difference can be relatively large and mass hiarchy $\left(M_{A}-M_{H}\right)$ is inverted (usually you find this difference negative, where as in this case it is positive), and production cross secton for $\mathrm{A}$ and $\mathrm{H}$ are large.

\section{DISCUSSION}

An upgrade from a neutrino factory to a Muon collider Higgs factory is under investigation. In addition to being costly, it would require more cooling, acceleration etc. Additional studies and cooling demonstration is necessary. The physics discovery poetential at a Higgs factory was presented. We illustrated special cases (low and high $M_{S U S Y}$ ) when the cross section become exactly zero, and corresponding regions of space were $\sigma_{M S S M}\left(\mu^{+} \mu^{-} \rightarrow h\right) / \sigma_{S M}\left(\mu^{+} \mu^{-} \rightarrow h\right)<30 \%$. This seems to indicate that, in the regions where $\sigma$ is zero (no resonant production even of the lightest Higgs-boson is possible), the Neutral MSSM Higgs sector via resonant production of $\mathrm{H}$ and A may be possible at a muon collider.

\section{REFERENCES}

[1] Z. Parsa, Neutrino Factory Based on Muon - Storage - Rings to Muon Colliders: Physics and Facilities, PAC2001.

[2] Z. Parsa and W. J. Marciano (eds.), Proc. Intersections of Particle and Nuclear PhysicsAIP CP 5491019 pages, NY (2000); Z. Parsa ed., Future High Energy Colliders AIP CP 397 (1997).

[3] A. Czarnecki, W. J. Marciano, hep-ph/0102122 (9 Feb 2001)\& refs.; H. N. Brown et al., Phys. Rev. Lett. 86, 2227 (2001); Private Comm:Lee Roberts (g-2) (5/01).

[4] S. Kuhlman et al., Physics Goals of the Next Linear Collider, BNL report 63158; ZDR Report for NLC, SLAC.

[5] C.M. Ankenbrandt etal, Phys. Rev. ST Accel. Beams 2, 081001 (1999),\& refs.; $\mu^{+} \mu^{-}$Collider Study, BNL-52503.

[6] V. Barger, M. Berger, K. Fujii, J. Gunion, T. Han, C. Heusch, W. Hong, S. Oh, Z. Parsa, S. Rajpoot, R. Thun, W. Willis, in AIP CP 352 ed. D.B. Cline (1996).

[7] Z. Parsa, Polarization and Luminosity requirements for the First Muon Collider, in AIP Conf. Proc. 472, pp. 251-259.

[8] Private comm.: S. Heinemeyer, figs2-4;BNL-HET01/22.

[9] NUF Study II: www.cap.bnl.gov/mumu/studyii; Study I: www.fnal.gov/projects/muon_collider/nu_factory/; Z. Parsa, Muon Storage Rings - Neutrino Factories, AIP CP 533, pp. 181-195, N.Y. (2000) \& Refs. therein. 